\title{
Flexibilidad de la jornada laboral para aumentar la productividad, Lima 2016-2018
}

\section{Flexible working day to increase productivity, Lima 2016-2018}

\author{
Luis Humberto Ludeña Saldaña* \\ Facultad de Ciencias Contables, Económicas y Financieras, \\ Universidad de San Martín de Porres, Perú
}

\section{Resumen}

En el Perú existe la necesidad de flexibilizar la jornada laboral como medida para reducir el desempleo, fomentar la productividad y mejorar la calidad de vida de los trabajadores. El ritmo de vida moderna hace que las personas distribuyan su tiempo entre el centro de trabajo y la familia, el problema es definir el límite de horas de trabajo y cuánto tiempo va a compartir con la familia. En el presente estudio se busca identificar la relación entre reducción de la jornada laboral y productividad del trabajo. Por consiguiente, se aplicó una encuesta para conocer la percepción sobre la reducción de la jornada laboral, resultando que $97 \%$ considera que las MYPES están cambiando casi al mismo ritmo que el resto de las empresas, y que para sobrevivir tienen que adaptarse muy rápido al cambio; asimismo, la flexibilidad de la jornada laboral está siendo aceptada por este sector. El 89\% considera que si se flexibiliza el horario de trabajo, la productividad aumentaría; solo $11 \%$ considera que no afectaría. Por otro lado, el primer efecto de las jornadas muy extensas es el agotamiento físico de los trabajadores que actúa como un factor en contra del aumento de la productividad. La flexibilidad laboral impacta en los trabajadores como en la organización, lo que permite crear un entorno propicio para la innovación.

Palabras clave: jornada laboral; flexibilización del mercado de trabajo; horario de trabajo

Este es un artículo Open Access bajo la licencia Creative Commons Atribución-NoComercial-Compartirlgual 4.0

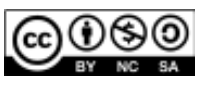




\begin{abstract}
In Peru, making working days more flexible is needed as a measure for reducing unemployment, promoting productivity and improving workers' quality of life. The pace of modern life leads people to balance their time between workplace and family: the issue is to establish a maximum number of working hours and how much time workers will share with their family. This research aimed at identifying the relationship between the reduction of working hours and work productivity. To that effect, a survey was conducted to find out how workers perceived the reduction of working hours: $97 \%$ believed that MYPEs (micro and small companies) are changing almost at the same rate as other types of companies, they have to adapt very quickly to changes in order to survive, and they are accepting flexible working days. Eighty-nine percent $(89 \%)$ considered that if working hours were flexible, productivity would increase; only $11 \%$ believed that productivity would not be affected. The first effect of extremely long working days is physical exhaustion of workers, a factor against productivity increase. Work flexibility has an impact on both workers and companies, since it enables the creation of a suitable environment for innovation.
\end{abstract}

Keywords: working day; labor market flexibilization; working hours

\title{
Introducción
}

Una persona natural distribuye su tiempo de vida (24 horas) entre el trabajo, el tiempo libre y el ocio, siendo el trabajo la actividad de carácter social y fundamental del hombre. «El trabajo es un deber y un derecho. Es base del bienestar social y un medio de realización de la persona» (Constitución Política del Perú, 1993, p. 40).

En la sociedad contemporánea el tiempo del trabajo puede venderse en el mercado laboral a un determinado precio, se le denomina salario y/o autoemplearse. El tiempo de trabajo vendido en el mercado laboral por medio de un contrato se denomina jornada laboral, en esta situación el trabajador no posee la propiedad ni el control sobre los frutos de su trabajo que se logren durante el periodo que dura la jornada. 
Al respecto, la Organización Internacional del Trabajo (OIT) sostiene:

El tiempo de trabajo, el descanso y la organización de las horas de trabajo y los períodos de descanso (la ordenación del tiempo de trabajo) son aspectos fundamentales de las relaciones laborales. La cantidad de horas trabajadas, la duración y número de períodos de descanso y la manera en que se distribuyen durante el día, la semana y el mes tienen importantes consecuencias tanto para los trabajadores como para los empleadores. (2019, p. 17)

El ritmo de vida moderno hace que el hombre distribuya su tiempo entre el centro de trabajo y la familia, el problema es definir el límite de horas de trabajo y cuánto tiempo va a compartir con la familia. A lo largo de la historia estos límites han variado, desde jornadas de trabajo esclavizantes con duración de más de 15 horas, hasta jornadas que están reglamentadas por los organismos internacionales como la OIT y las legislaciones nacionales. En el Perú, la duración de la jornada laboral de ocho horas diarias o 48 horas semanales está garantizada por la Constitución Política, sin embargo, también está socialmente aceptado que se puede establecer por ley, por convenio o decisión unilateral del empleador una jornada diferente a lo establecido legalmente.

El crecimiento económico siempre estuvo asociado a la productividad del trabajo, y esta, a la duración de la jornada laboral.

En la actualidad, la productividad es un concepto mucho más complejo, en el que entran en juego diferentes parámetros como la satisfacción laboral, la motivación, el clima organizacional, los valores, el trabajo en equipo. No se trata solo de cantidad de trabajo, sino de calidad del mismo. (Opere, 2019, p. 1)

La tecnología y la alta incidencia de los sistemas de información en todos los aspectos de la vida del hombre están originando una reevaluación del concepto de una jornada laboral rígida, se cuestiona la duración de ocho o más horas diarias y se postula por menos horas de trabajo. La mayor disponibilidad de tiempo libre es propicia para la creación de un escenario con mayores posibilidades para la innovación, porque el hombre en su rol de 
trabajador puede mejorar su capacitación y su capacidad de innovación. En su rol de consumidor tiene a su alcance y a bajo costo Internet, cable, etc. que le permite estar mejor informado y ser más exigente en los satisfactores de sus gustos y preferencias, que al mismo tiempo son muy cambiantes.

Por otra parte, las empresas se ven obligadas a innovar, sus requerimientos de mano de obra giran para un tipo de trabajador con otras capacidades y habilidades vinculadas a la creatividad e innovación, lo cual significa evaluar qué tan beneficioso es mantener sistemas rígidos de la jornada laboral, que no garantizan la plena utilización del tiempo de trabajo. Existe el riesgo de que ocurra tiempo laboral muerto. Como dice Opere (2019):

Una jornada laboral larga no es una garantía de mayor productividad porque existen trabajadores que pasan más horas de las estipuladas en el horario de trabajo sin que sean realmente productivos, mientras que otros pueden limitarse a sus horas asignadas y generar resultados superiores con una mejor gestión del tiempo. (p. 1)

Esta crítica, abierta a la rigidez de la jornada laboral, demanda una respuesta en torno a la flexibilidad en términos de duración y gestión de los horarios o los turnos.

Se plantea entonces que trabajadores y empresarios respondan con nuevos conocimientos y habilidades por un lado, y por otro, con una visión de futuro que anticipen la dirección e intensidad de los cambios, lo que demanda mayor disponibilidad de tiempo diferente a la duración legal de la jornada laboral.

La flexibilidad como tendencia en el mercado laboral está siendo vista como alternativa para mejorar la productividad, que sigue siendo un problema permanente como factor subyacente del crecimiento económico al mismo tiempo que un riesgo para aumentar el desempleo o subempleo. Al respecto, la OIT (2019) afirma:

En el 2018 había 172 millones de personas desempleadas en el mundo, una tasa de desempleo del 5,0 por ciento [...] Aparte de las personas desempleadas, hubo otros 140 millones de personas en la categoría de «fuerza de trabajo potencial», un grupo clasificable como fuerza de trabajo subutilizada. En este grupo de quienes buscan empleo pero no 
están disponibles para incorporarse a un empleo, o que están disponibles pero no buscan empleo, hay muchas más mujeres (85 millones) que hombres (55 millones). (p. 2)

Considerando las condiciones actuales del crecimiento económico, el desempleo se mantendrá a nivel mundial y dado que la mayoría de la población depende de su salario como ingreso principal, la pobreza seguirá afectando la calidad de vida de los trabajadores.

Entre las estrategias más importantes para disminuir el desempleo se encuentra la propuesta de la flexibilización del mercado laboral.

Los viejos y nuevos clásicos sostienen que el mercado laboral es flexible lo que significa que ese mercado se ajusta rápidamente a los cambios del entorno laboral. En otras palabras, los trabajadores siempre están dispuestos a recibir un salario menor, en épocas de contracción económica, a su vez los empresarios estarán interesados en incrementar el salario si los trabajadores así lo exigen. Esto los llevó a pensar que si existe desempleo es porque los individuos no quieren trabajar a ese salario que le ofrece el empresario. Este razonamiento, también los llevó a concluir, que el desempleo es voluntario ya que el trabajador conscientemente no acepta el trabajo por el salario ofrecido. (Menco, marzo de 2008, p. 1)

Por consiguiente, si el salario baja la demanda de trabajo aumentará y se podrán emplear a más trabajadores. Mayor cantidad de personas dispondrán de un ingreso lo que significa mayores posibilidades para satisfacer sus necesidades. Se presume que ante la disminución del salario los costos de producción bajen, en este nuevo escenario se espera que la demanda se incremente y esta se convierta en un nuevo impulso sobre la inversión y la economía reinicie el proceso de crecimiento, resultando la flexibilidad laboral un impulso para la economía como sistema.

Con la hegemonía de la flexibilidad del mercado y las transformaciones que actualmente están sucediendo por los avances y las mejoras en la tecnología y las comunicaciones, que perturban e incluso acentúan la eliminación de muchos aspectos tradicionales relacionados con la dimensión temporal y 
espacial del trabajo (OIT, 2019), la regulación del tiempo de trabajo resulta aún más importante.

La OIT (2019) considera la flexibilidad del mercado laboral como una alternativa para que las empresas puedan disponer de mayores posibilidades con el objetivo de aumentar la movilidad en las relaciones laborales que genera con sus trabajadores. Así mismo, Arancibia (2011) menciona que la flexibilidad del mercado laboral puede ser interna y externa, ambas con sus propios componentes o subdimensiones.

Flexibilidad interna o flexibilidad en la regulación de las condiciones de trabajo es definido por Arancibia (2011):

Como la capacidad otorgada por la legislación laboral, a las empresas, para celebrar contratos individuales de trabajo que permitan variar elementos específicos de las condiciones laborales dentro de los márgenes permitidos por la ley. La flexibilidad interna estaría compuesta por: la flexibilidad salarial, la flexibilidad en los horarios de trabajo, la flexibilidad en la jornada de trabajo y la flexibilidad funcional. (p. 45)

Es importante remarcar que la flexibilidad en los horarios de trabajo es entendida como «la disposición variable, por parte de la empresa, de las horas de trabajo que deben ejecutar sus empleados en la misma» (Arancibia 2011, p. 45).

De esta forma, las horas de trabajo que un empleado debe laborar dependerán de la demanda de bienes y servicios que tenga en determinado momento la empresa para la cual trabaja.

La empresa contratará más horas de trabajo cuando tenga que satisfacer una demanda creciente de los bienes y servicios que produce; reducirá las horas de trabajo de su personal cuando la demanda se contrae, eliminando de esta forma horas de trabajo innecesarias.

De la flexibilidad de la jornada laboral que se origina por las fluctuaciones de la demanda se desprende que el uso del trabajo puede gestionarse por turnos los fines de semana, y así redistribuir mejor los permisos y descansos anuales en armonía con las necesidades de los trabajadores. 
La flexibilización externa es entendida como la libertad que otorga la legislación laboral de un país a las empresas para modificar la cantidad de sus trabajadores, por medio de nuevas modalidades de contratación de fuerza de trabajo diferentes al contrato de duración indefinida. Estas modalidades de contrato le otorgan márgenes de libertad a la empresa para racionalizar el número de trabajadores y horas de trabajo, de acuerdo a sus necesidades con mayor agilidad y menos costos.

La OIT (2019) señala:

Largas jornadas laborales reducen claramente el tiempo disponible para la vida familiar y muchas veces requieren trabajo en las noches y los fines de semana. Sin embargo, muchos trabajadores laboran horas extra para poder generar un aumento del ingreso familiar. Por otra parte, para quienes se desempeñan en cargos gerenciales y profesionales, trabajar muchas horas diarias puede ser visto como una forma de demostrar el compromiso y progresar en la organización. (p. 1)

Desde la perspectiva del taylorismo, para aumentar la productividad laboral se requiere tener control del tiempo de trabajo, posición que todavía se mantiene y en muchas empresas de diversos sectores permanece la tendencia a la prolongación de la jornada laboral más allá de las ocho horas con poca importancia por la productividad marginal. Como sostiene Pozen, «los empleados de cuello blanco están trabajando más que nunca. Muchos llegan temprano a la oficina, se van tarde, trabajan durante el almuerzo y permanecen en sus escritorios los fines de semanas. Sin embargo, estos trabajadores son mínimamente productivos» $(2018$, p. 1).

\section{Problema}

A finales de la segunda década del siglo XXI una de las preocupaciones fundamentales para reavivar el crecimiento económico y mantener el impulso del progreso social es impulsar la productividad mediante el empoderamiento de todos los trabajadores y el desencadenamiento de la innovación y del dinamismo emprendedor, situación que está vinculada a la duración de la jornada laboral. 
Entender la estrecha relación entre la productividad, la duración de la jornada laboral y su incidencia en el crecimiento y desarrollo de las sociedades, es un tema de preocupación y discusión para la academia como para los empresarios porque «la organización [...] no es una simple máquina [...] por encima de todo, es un ente social. Es un grupo de personas. Su objetivo [...] tiene que ser que los puntos fuertes de las personas sean eficaces y sus debilidades irrelevantes» (Drucker, como se citó en Cage, 2016)

La productividad tiene que ver con trabajar de forma más inteligente más que con «trabajar más arduamente» y refleja la capacidad de generar más resultados o productos mediante una mejor combinación de los insumos, gracias a nuevas ideas, innovaciones tecnológicas y nuevos modelos de negocios. (Organización para la Cooperación y el Desarrollo Económico - OCDE, 2015, p. 1)

Esto quiere decir que tener una jornada laboral continua, no es una garantía de mayor productividad. Existen países como el Perú, donde las jornadas de trabajo son prolongadas, por ejemplo, es muy común un horario laboral que se extiende desde las 08:00 horas hasta más de las 17:00 horas, con un pequeño descanso para el almuerzo, alrededor de media hora; en estos países la productividad es menor que la de los países de menor duración de la jornada. Estos trabajadores menos productivos disponen de menos horas para compartir en familia o mejorar sus habilidades y conocimientos necesarios para iniciar un proceso de innovación.

Por lo tanto, resulta importante encontrar un punto de equilibrio entre el tiempo destinado a la vida personal, familiar y laboral lo que incide directamente en la felicidad de los trabajadores.

La propuesta de encontrar el punto de equilibrio entre el tiempo destinado a la vida personal, familiar y laboral en el presente siglo es defendida por la Asociación para la Racionalización de los Horarios Españoles (ARHOE), que por intermedio de su vocero Casero (2 de mayo de 2016) plantea:

La racionalización de horarios es un factor clave para aumentar la eficacia y la productividad en el ámbito laboral. Necesitamos que nuestros sistemas de trabajo evolucionen rápidamente hacia unos 
objetivos de mayor calidad, y para ello es fundamental aplicar estrategias de motivación, estimular un pensamiento positivo frente a la adversidad, buscar fórmulas que incrementen el rendimiento de los trabajadores o liberar la mente de estrés para trabajar mejor.

También, hace una amplia crítica por el uso prolongado de horas y horas de trabajo que muchas veces son improductivas.

Por otro lado, existen experiencias en países europeos como Francia donde la duración legal de la jornada laboral es de 35 horas semanales; no obstante, esta no es una duración máxima, es un promedio.

[En] la práctica las empresas pueden realizar un horario superior. La diferencia entre el total de las horas realizadas y las 35 horas legales se compensa de manera compleja. Los trabajadores tienen derecho a dos días y medio laborables de vacaciones por mes trabajado. Las vacaciones se contabilizan desde el 1 de junio del año anterior, hasta el 31 de mayo del año en curso, es decir 30 días hábiles (como se cuentan los sábados, corresponden a cinco semanas) por 12 meses de trabajo efectivo. Es preciso señalar que son numerosos los convenios que mejoran la situación. (Moncat.gencat.cat, 2019)

Tabla 1

Europa, duración de la jornada laboral en horas semanales 2011-2018

\begin{tabular}{lcccccccc}
\hline & 2011 & 2012 & 2013 & 2014 & 2015 & 2016 & 2017 & 2018 \\
\hline Países Bajos & 31 & 31 & 31 & 31 & 31 & 31 & 31 & 31 \\
Alemania & 35 & 35 & 35 & 35 & 35 & 35 & 35 & 35 \\
Francia & 36 & 35 & 35 & 35 & 35 & 35 & 35 & 35 \\
Irlanda & 34 & 34 & 34 & 34 & 34 & 34 & 35 & 35 \\
España & 37 & 36 & 36 & 36 & 36 & 36 & 36 & 36 \\
Estados Unidos & 36 & 37 & 37 & 37 & 37 & 37 & 37 & 37 \\
Luxemburgo & 38 & 38 & 38 & 38 & 38 & 38 & 38 & 38 \\
Suecia & 36 & 35 & 35 & 35 & 35 & 36 & 35 & 35 \\
Reino Unido & 35 & 35 & 36 & 36 & 36 & 36 & 36 & 36 \\
Suiza & 37 & 36 & 36 & 36 & 36 & 36 & 36 & 36 \\
\hline
\end{tabular}

Nota: Datos de las estimaciones modeladas de la OIT (2019). 


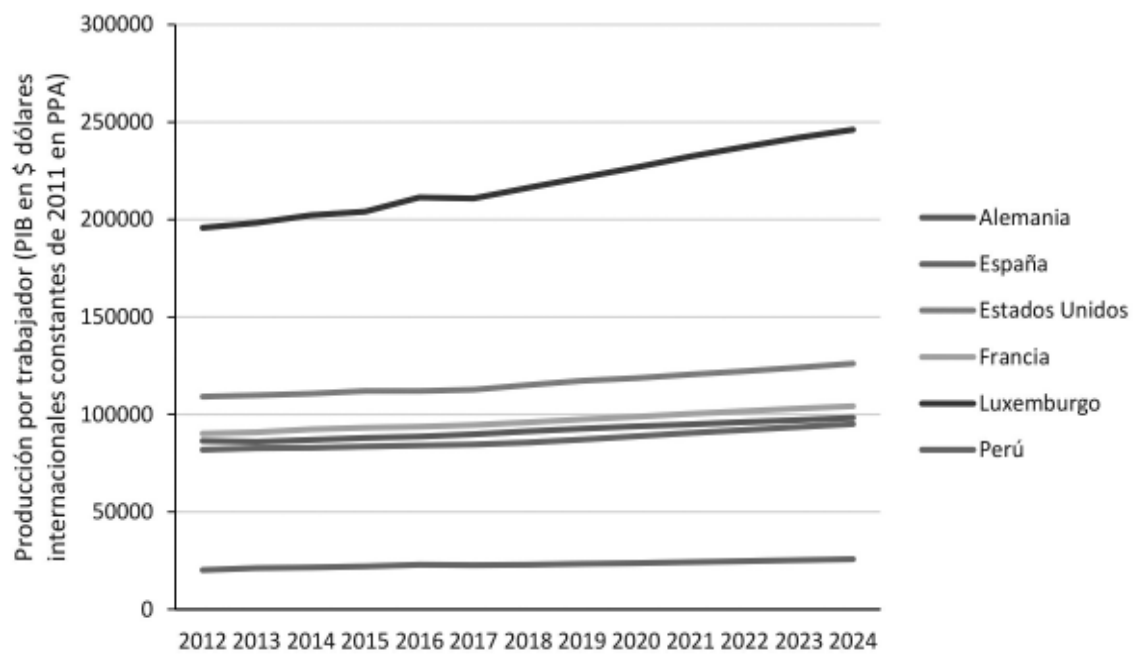

Figura 1. Productividad de los cinco países más productivos del mundo 2012-2024.

Fuente: Estimaciones modeladas de la OIT (2019).

Alemania, con jornadas de 35 horas semanales en promedio, evidencia ser más eficaz y productivo en sus trabajos; en la Tabla 2 se observa que durante el periodo 2012-2018 la jornada laboral en promedio fue de 35 horas semanales y la productividad muestra una tendencia creciente.

\section{Tabla 2}

Alemania, productividad y jornada laboral 2012-2019

\begin{tabular}{lcccccccc}
\hline \multicolumn{1}{c}{ Alemania } & 2012 & 2013 & 2014 & 2015 & 2016 & 2017 & 2018 & 2019 \\
\hline $\begin{array}{l}\text { Productividad } \\
\begin{array}{l}\text { Producción por } \\
\text { trabajador }\end{array}\end{array}$ & 86373 & 85870 & 86898 & 87942 & 88612 & 89748 & 91358 & 92718 \\
$\begin{array}{l}\text { (PIB en \$ dólares } \\
\text { internacionales } \\
\begin{array}{l}\text { constantes de 2011 } \\
\text { en PPA) }\end{array}\end{array}$ \\
$\begin{array}{l}\text { Jornada laboral } \\
\text { (horas semanales) }\end{array}$ & 35 & 35 & 35 & 35 & 35 & 35 & 35 & 35 \\
\hline
\end{tabular}

Fuente: Estimaciones modeladas de la OIT (2019).

En Suecia se realizó un experimento para probar la efectividad de la jornada laboral de seis horas en una residencia de ancianos de Gotemburgo. 
Asegura que la jornada laboral de seis horas es demasiado costosa. Durante dos años, este centro ha permitido a las 68 enfermeras trabajar dos horas menos de lo que es usual y mantener el mismo sueldo. La medida mejoró la calidad de vida de los trabajadores, mejorando la salud de los mismos y, por tanto, el número de bajas. Pero para compensar el menor número de horas trabajadas, tuvieron que contratar a otros 17 empleados. Estas contrataciones se tradujeron en un sobrecoste de 12 millones de coronas suecas, lo que equivale a 1.260.000 euros. (La Información, 4 de enero de 2017, p. 1)

El resultado de esta experiencia en el grupo de trabajadoras condujo a la reducción del ausentismo laboral y las actividades que llevaron a cabo con los residentes aumentaron en un $64 \%$. Además, al considerar el aspecto emocional, afirmaron sentirse más felices. A partir de los resultados en este experimento se estableció que la productividad y la felicidad del trabajador están asociadas, es decir, con trabajadores felices se logra mayor productividad.

En otros países también se ha logrado mejorar la productividad al reducir la jornada laboral, tal es el caso de Rusia donde en promedio la jornada es de 40 horas (Moscú), en los profesionales -en algunos casos- la jornada es de 39 horas, además la alta productividad ha conducido a mejorar sus salarios. De esta experiencia se deduce que mayores salarios estarían vinculados a la mayor productividad. Según Datosmacro.expansion.com (2018) el salario mínimo interprofesional para el 2018 en Rusia quedó fijado en 152.8 al mes, vale decir 1834 euros al año, teniendo en cuenta que se consideran 12 pagas anuales. En la Figura 2 se muestra la tendencia creciente del salario mínimo durante los últimos ocho años.

En América Latina, países como Panamá, Argentina y Chile registran la tasa de crecimiento de productividad más alta. En estos países se ha reducido la jornada laboral; Argentina desde el año 2012 tiene una jornada laboral promedio de 38 horas semanales, al 2018 evidenció 37 horas; Panamá, en el año 2012, de una jornada laboral de 43 horas fue bajando paulatinamente hasta que en el 2018 tuvo una jornada de 38 horas semanales; Brasil se mantiene en jornadas laborales de 39 horas semanales (Tabla 3). Países como México, Ecuador y Brasil que tienen las jornadas laborales más extensas presentan tasas de crecimiento de la productividad muy baja. 


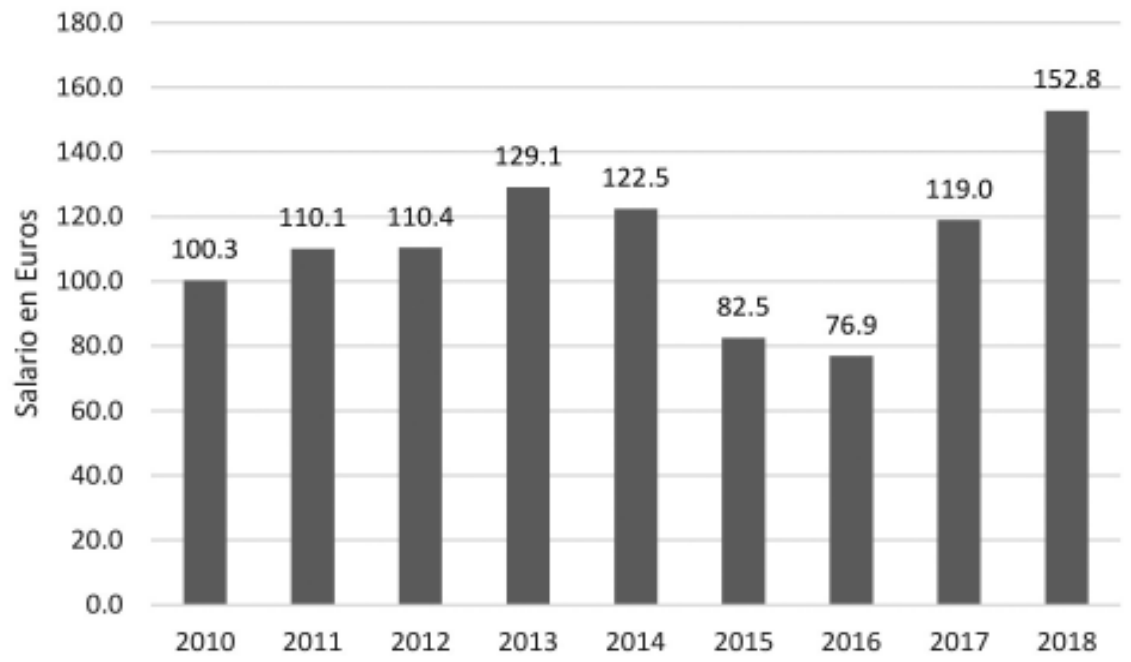

Figura 2. Rusia, salario mínimo interprofesional 2010-2018 en euros.

Fuente: Elaboración propia a partir de https://datosmacro.expansion.com/smi/rusia

Tabla 3

América Latina: Tasa de crecimiento de la productividad 2012-2018

\begin{tabular}{lccccccc}
\hline País & 2012 & 2013 & 2014 & 2015 & 2016 & 2017 & 2018 \\
\hline Panamá & 4.4 & 3.2 & 4 & 3.4 & 2.5 & 2.3 & 3.7 \\
Argentina & -2 & 1.6 & -2.6 & 1.4 & -3 & 1.6 & 3.1 \\
Chile & 3.3 & 2 & 0.6 & 0.8 & 0.5 & 0.2 & 2.9 \\
Bolivia & 6.9 & 4.2 & -0.1 & 10.3 & -3.1 & 6.4 & 1.6 \\
Colombia & 0.7 & 3.1 & 2.3 & 0.8 & 1.6 & 1.1 & 1.3 \\
Brasil & -0.5 & 1.4 & -0.9 & -3.3 & -0.9 & 0.7 & 1 \\
El Salvador & -0.3 & 0.1 & 3.1 & 2.2 & 0.6 & -1.1 & 0.9 \\
Perú & 4.1 & 4.9 & 1.4 & 3.1 & 3.7 & -1.2 & 0.9 \\
Ecuador & 2.2 & 3.6 & 2.5 & -5.3 & -5.2 & -0.7 & 0.4 \\
México & 0.1 & -0.3 & 1.7 & 0.6 & 0.7 & 0 & 0.2 \\
Venezuela, & & & & & & & \\
República & & & & & & & \\
Bolivariana & 4.2 & 0.4 & -6 & -7.1 & -9.8 & -9.7 & -7.3 \\
\hline
\end{tabular}

Fuente: Estimaciones modeladas de la OIT (2019).

Indicador ODS 8.2.1 - Tasa de crecimiento anual de la producción por trabajador (PIB en dólares internacionales constantes, PPA 2011) (\%). 
La tasa de crecimiento anual de la productividad laboral se muestra en la Tabla 3 y 4, la misma que representa el volumen total de producción por una unidad laboral (Producto Interno Bruto, PIB/ cantidad de personas ocupadas). Evidencia que países con jornadas más largas han mostrado una caída en su productividad como en algunos países de América Latina (Panamá, México, Chile); países con la jornada laboral más corta son más productivos (Alemania, Francia, España), situación que está altamente relacionada con la calidad y la eficiencia del trabajo humano (Tabla 4).

Tabla 4

América Latina: duración de la jornada laboral por horas semanales 2010-2018

\begin{tabular}{lccccccccc}
\hline País & 2010 & 2011 & 2012 & 2013 & 2014 & 2015 & 2016 & 2017 & 2018 \\
\hline Argentina & 40 & 40 & 39 & 39 & 39 & & & 38 & 37 \\
Panamá & & 44 & 43 & 43 & 43 & 43 & 42 & 42 & 38 \\
Brasil & & & 40 & 40 & 40 & 39 & 39 & 39 & 39 \\
Chile & & & & & & & & 42 & 41 \\
México & & & & 46 & 46 & 46 & 47 & 47 & 47 \\
\hline
\end{tabular}

Fuente: Estimaciones modeladas de la OIT (2019).

Sobre la duración de la jornada de trabajo existen ideas preconcebidas que conllevan a diversas formas de mirar la realidad. Por ejemplo, desde una visión aislada que no toma en cuenta los factores socioambientales donde el trabajador se encuentra inmerso, por el lado del empresario, sostienen que con una reducción de la jornada de trabajo se va a producir menos; mientras que por el lado del trabajador, piensan que les van a pagar menos. Desde esa perspectiva, puede que el bienestar personal guarde una débil relación con la cantidad de horas trabajadas. También ocurren jornadas cortas de trabajo con alta presión que originan cuadros de estrés y fatiga en los trabajadores, afectando negativamente a la productividad y a la integridad. En general, jornadas muy largas o cortas que conduzcan al agotamiento físico de las personas impactarán negativamente en la productividad.

Con una visión más sistémica del trabajador en su entorno, las jornadas cortas con un ambiente laboral agradable, más colaborativo y de menos confrontación serán generadoras de un clima propicio para la innovación, las personas se sentirán más motivadas y relajadas para trabajar. 
Por ende, resulta que no basta con disminuir o aumentar la jornada laboral independiente de los factores socioambientales, sino cambiar las relaciones laborales como parte de la vida del hombre; así como optar por un modelo de producción donde uno de sus ejes sea construir relaciones laborales empresatrabajador que tengan por objetivo simultáneamente satisfacer las necesidades de los trabajadores sin descuidar las metas y demandas de la empresa.

\section{La verdad versus lo que se piensa}

El futuro del mercado laboral está siendo impactado por las TIC. Debido al «surgimiento de los empleados digitales, programados con inteligencia artificial se conjetura que abrirán la puerta a una semana laboral de solo tres jornadas» (Gestión, 9 de mayo de 2019). De aquí nace la preocupación por considerar que es vital cambiar el modelo de la relación empleado y empleador, promoviendo un tipo de trabajo con objetivos comunes que se convierta en el eje central para aumentar la productividad y al mismo tiempo disminuir la pobreza y la desigualdad; y que esa vieja relación empleadoempleador adquiera significados de nuevo tipo de relación laboral con más flexibilidad donde los objetivos sean colectivos y se garantice la sostenibilidad de desarrollo.

Por otra parte, la presencia de un personal técnicamente capacitado en la empresa otorga seguridad y promueve mayores y mejores relaciones laborales; asimismo, permite la eliminación paulatina de viejos paradigmas relacionados con horarios de trabajo, lo que hace posible enrumbarse hacia el cumplimiento de metas. La flexibilidad de esta manera, conlleva a la autogestión empresarial y a que sus integrantes avancen en función de cumplir objetivos mayores.

En tiempos actuales se aprecia una tendencia en las organizaciones líderes, y en algunos países a nivel mundial, a considerar la flexibilización de la jornada laboral como una alternativa para incrementar la productividad y mejorar la calidad de vida de los trabajadores.

En un contexto de constante cambio, en un mundo moderno y tecnológico, resulta pertinente considerar como objetivo analizar la relación entre la jornada laboral y la productividad del trabajo desde la percepción de los líderes de las organizaciones en Lima. 


\section{Método}

\section{Participantes}

La muestra la integran 35 líderes (Figura 3) de una población compuesta por líderes (gerentes) del área laboral de las empresas del sector público y otras organizaciones empresariales.

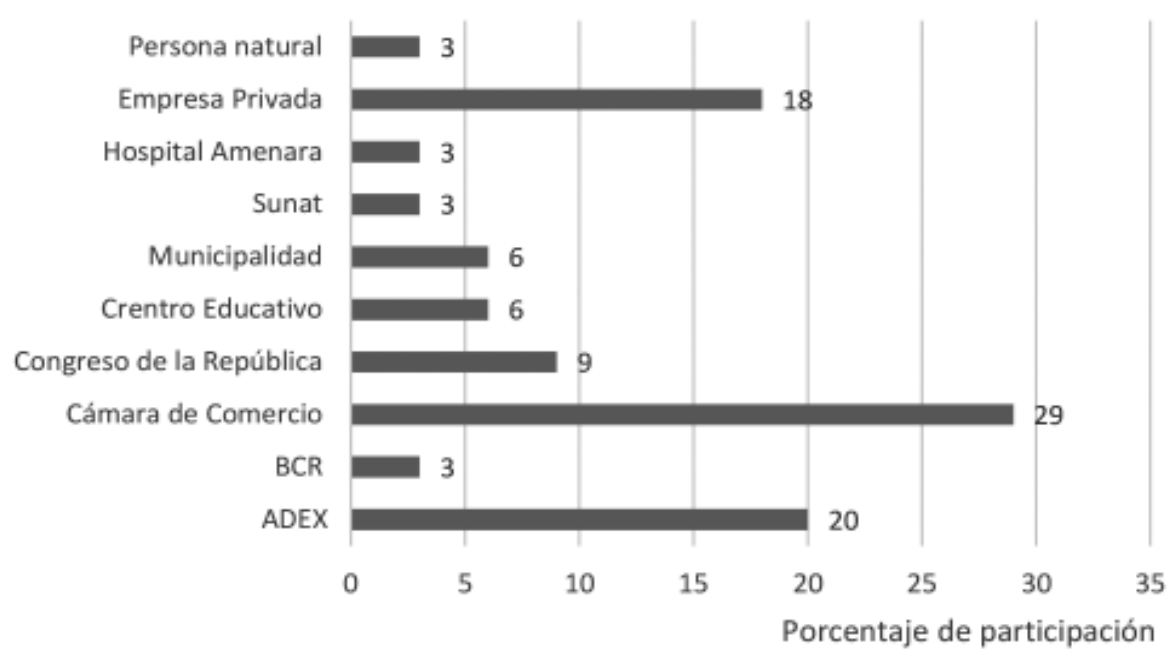

Figura 3. Composición de la muestra.

Fuente: Elaboración propia con datos de la muestra.

\section{Instrumento}

Se utilizó un cuestionario para explorar la percepción y opinión de los líderes vinculados al aparato productivo acerca de la influencia de la flexibilidad laboral en la productividad de la mano de obra.

\section{Procedimiento}

Inicialmente se realizó una revisión de la información secundaria de las investigaciones y estadísticas del INEI. Posteriormente, se aplicó el cuestionario. El periodo de observación se desarrolló en Lima Metropolitana, entre los años 2015-2018. 


\section{Resultados}

\section{Duración de la jornada laboral y productividad}

En América Latina desde hace más de 20 años se viene discutiendo sobre la necesidad de flexibilizar el mercado de trabajo con el objetivo de reducir el alto desempleo, fomentar la competitividad del aparato productivo, hacer mejor uso del capital humano y dotar a la economía de mayor dinamismo. En el Perú se han llevado a cabo una serie de reformas laborales desde la década de 1990; con las primeras reformas se flexibilizaron los sistemas de contratación laboral, pero significó también el aumento de los despidos masivos, acompañados por un debilitamiento de las instituciones laborales como los sindicatos.

Sin embargo, los resultados de las sucesivas reformas hasta el momento son muy controversiales, en algunos casos tuvieron efectos negativos en el mercado de trabajo, por ejemplo, el uso intensivo de la contratación a plazo fijo o temporal, tanto en el sector público como en el privado.

Por otro lado, la tasa de actividad muestra una tendencia decreciente reflejando un menor grado de participación de la población en edad de trabajar en la actividad económica y se calcula relacionando la población económicamente activa, con la población en edad de trabajar (Figura 4). En el año 2007 la tasa era de 73.8 y al 2017 ha descendido a 72.4

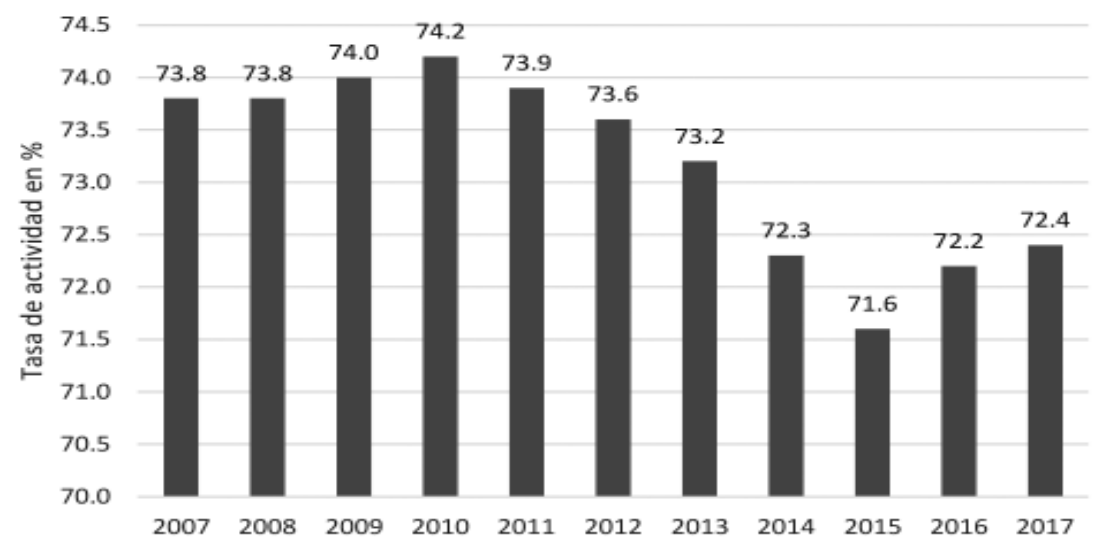

Figura 4. Perú: Tasa de actividad 2007-2017.

Fuente: Elaboración propia con datos del INEI, 2018 
El Perú, como algunos países de América Latina, registra las jornadas laborales más extensas, entendiendo esta como el tiempo que el trabajador está a disposición del empleador para brindar el servicio sobre la base de un contrato firmado, que en algunos casos es menor que el horario de trabajo (es la medida de la jornada que determina con exactitud la entrada y salida en cada día de trabajo, por ejemplo, la jornada laboral es de 8 horas pero el horario de trabajo es de 12 horas). En algunos sectores la era tecnológica ha dado pie a que, en muchas ocasiones, los horarios de trabajo se alarguen más de lo previsto porque el trabajador puede retirarse del lugar de trabajo, pero sigue manteniendo contacto con el jefe mediante los correos electrónicos y el wasap para dar o recibir mensajes.

La legislación peruana regula la jornada laboral tal como se señala en el Decreto Legislativo $N^{\circ} 854$ (El Peruano, 1996), Ley de jornada de trabajo, horario y trabajo en sobretiempo:

La jornada ordinaria de trabajo para varones y mujeres mayores de edad es de ocho (8) horas diarias o cuarenta y ocho (48) horas semanales como máximo. Se puede establecer por ley, convenio o decisión unilateral del empleador una jornada menor a las máximas ordinarias.

En el mismo Decreto Legislativo, se señala en su artículo 6:

Es facultad del empleador establecer el horario de trabajo, entendiéndose por tal la hora de ingreso y de salida, sin perjuicio de lo establecido en el Artículo 2, inciso (d). Igualmente está facultado a modificar el horario de trabajo sin alterar el número de horas trabajadas. Si la modificación colectiva del horario es mayor a una hora y la mayoría de los trabajadores no estuviera de acuerdo, podrán acudir a la Autoridad Administrativa de Trabajo para que se pronuncie sobre la procedencia de la medida en un plazo no mayor de diez (10) días hábiles, en base a los argumentos y evidencias que propongan las partes. La resolución es apelable dentro del tercer día. Si la modificación tiene carácter individual, la impugnación de la medida por el trabajador se efectuará conforme a las disposiciones de la Ley Orgánica del Poder Judicial. 
En la realidad laboral de cada día, el empleador puede extender la jornada más allá de la máxima legal, como en los centros de trabajo informales, la jornada laboral es mayor de 8 horas diarias o 48 horas semanales y en muchos casos sin incrementar la remuneración. En los centros de trabajo formales se observa también el incremento de la jornada diaria, ocurre que a veces las horas extras son pagadas junto con la remuneración o en algunos casos son compensadas con descansos, previo acuerdo entre el trabajador y el empleador en un tiempo equivalente al trabajado.

Esta situación no se refleja en una mayor productividad, no nos encontramos ni cerca de estar entre los países más productivos. Como se observa en la Figura 5 las zonas donde la jornada de trabajo dura más hay menos productividad, es el caso de Iquitos o Juliaca, zonas de climas fuertes.

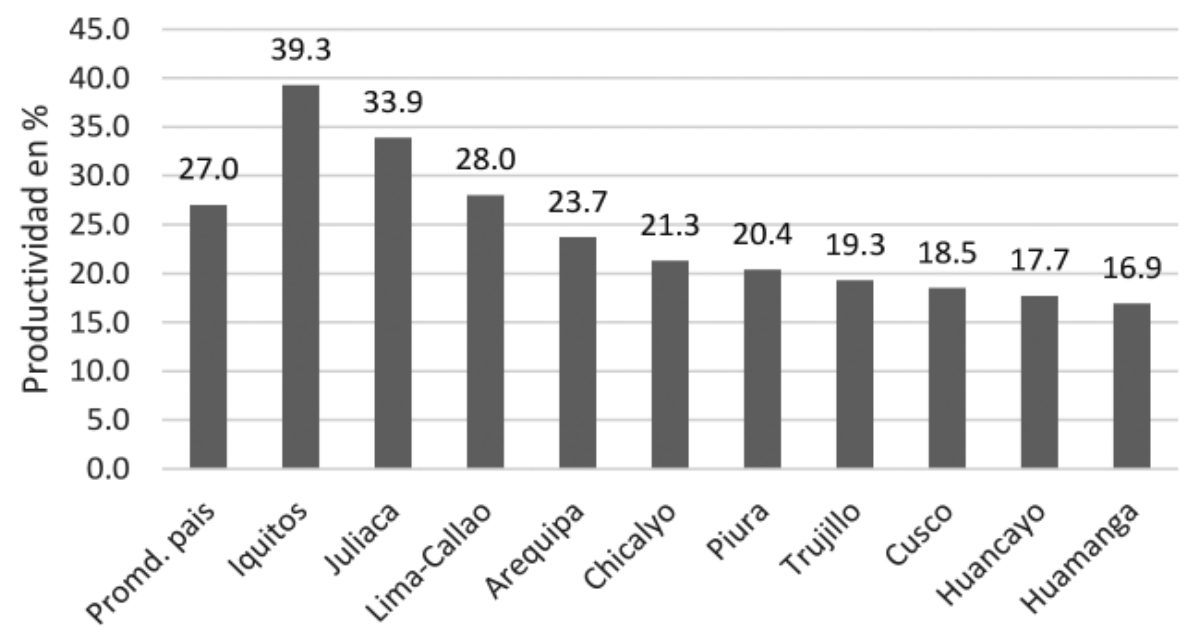

Figura 5. Perú: productividad del trabajo de la micro y pequeña empresa, 2012.

Fuente: Datos de INEI, 2018.

Por ello, es imprescindible conocer la percepción de los actores relacionados con la flexibilidad laboral, pues existen posturas y concepciones marcadamente diferentes en torno a su significado y a sus consecuencias.

En la muestra -a la que se le aplicó la encuesta- el 69\% eran trabajadores con jornada laboral completa de 48 horas semanales; $22 \%$ trabajadores a tiempo parcial y $8 \%$ que trabajaban más de la jornada de 8 horas diarias. 


\section{Jornada laboral y la flexibilización de los horarios}

En América Latina existe al 2019 una tendencia significativa para aplicar la flexibilidad laboral en los centros de trabajo, la percepción de la muestra coincide con esto. El 36\% considera necesario flexibilizar la jornada para mejorar la productividad; $31 \%$ optó por flexibilizar el horario de trabajo, mientras que $22 \%$ por flexibilizar considerando la naturaleza de los puestos de trabajo; y 11\% consideró incorporar el trabajo a distancia utilizando los medios tecnológicos existentes en el mercado.

\section{Flexibilidad laboral en las MYPES}

Existe una verdad incuestionable que no podemos obviar y es el hecho que en la actualidad son las MYPES en el Perú las que tienen una alta incidencia en la generación de puestos de trabajo dentro de la estructura productiva, representan el 94\% del universo de las empresas manufactureras (INEI, 2018). Y existe una marcada heterogeneidad en cuanto a la distribución geográfica, concentrando la ciudad de Lima un 19\% del total (217 553) en las cuales la presencia de la mujer es sobresaliente (Ministerio de la Producción, 2017).

Su importancia es evidente para el futuro de la economía del país. De acuerdo con las encuestas el $97 \%$ considera que las MYPES están cambiando casi al mismo ritmo que el resto de las empresas, para sobrevivir tienen que adaptarse muy rápido al cambio, de allí que la flexibilidad de la jornada laboral también está siendo aceptada por este sector.

Tal como se evidencian en las encuestas, frente a la pregunta icree usted que las medidas de flexibilidad laboral se están acentuando cada vez más en las MYPES?, 97\% contestaron que sí se están acentuando, frente a 3\% que dice no acentuarse la tendencia.

\section{Flexibilización de los horarios de trabajo y productividad}

Trabajadores sanos, motivados con todas sus capacidades, producen más y más rápido obteniéndose mayor productividad; jornadas muy largas conducen al agotamiento físico y mental de los trabajadores por la falta de descanso entre una jornada y otra, originando problemas de salud a mediano plazo como stress, ansiedad o trastornos que requieren tratamientos 
prolongados que afectan necesariamente el rendimiento laboral con efectos negativos en la productividad.

Desde la percepción de la muestra el $89 \%$ considera que si se flexibiliza el horario de trabajo la productividad aumentaría, mientras solo un $11 \%$ considera que no.

\section{¿Qué opina si se reduce la jornada laboral a cinco horas?}

En un entorno donde la cultura del trabajo premia la alta cantidad de horas en un puesto laboral, reducir a cinco horas la jornada laboral sin modificar el sueldo, produciría espanto en el empresario, sin embargo, experiencias de otras realidades muestran que existen mejores oportunidades para que el trabajador pueda conciliar su vida laboral con la vida personal, la motivación por el trabajo aumenta, se genera un ambiente laboral positivo. El hecho de reducir la jornada laboral hará que los empleados se ayuden y colaboren entre ellos para alcanzar los objetivos.

Tal como se evidencia en la encuesta el $52 \%$ de los encuestados considera que sería muy favorable y $31 \%$ favorable, solo un $17 \%$ sigue pensando que no sería favorable.

Son beneficios esperados a partir de la flexibilidad laboral (Tabla 5):

- Aumenta la productividad y se produce una reducción de costos, mejor gestión del horario de trabajo, se eliminan reuniones innecesarias, organizan y priorizan tareas (29\%).

- La conflictividad en el centro de trabajo se reduce. Producto de la reducción de la jornada laboral los empleados se ayudan y colaboran entre ellos para alcanzar los objetivos, creando un ambiente de trabajo positivo (43\%).

- Existe una mejor gestión del horario, reducción de ausencias y bajas laborales $(23 \%)$.

- Mejor uso del tiempo libre (5\%). 
Tabla 5

Beneficios esperados de la flexibilidad laboral

\begin{tabular}{lc}
\hline \multicolumn{1}{c}{ Categorías } & En \% \\
\hline Aumento de la productividad y una reducción de costos & 29 \\
Reduce la conflictividad en las empresas & 43 \\
Pueden organizar mejor sus horarios & 23 \\
Pueden usar mejor su tiempo libre & 5 \\
\hline Total & 100 \\
\hline
\end{tabular}

Fuente: Encuesta.

\section{Efectos negativos de la reducción de la flexibilización laboral}

En la última década algunos empresarios, así como autoridades de diversos países han realizado intentos por reducir la jornada laboral. En Suecia, en la ciudad de Gotemburgo se llevó a cabo un experimento -la casa de Volvo- en el que los resultados evidenciaron que los costos superaron los beneficios. Asimismo, en Corea del Sur, se encontró que los empleados valoran los días laborales cortos solo en la teoría (Journal of Happiness Studies). Mientras que en la OCDE se concluyó que cuanto más trabajaban las personas, más disminuía la productividad (The Economist) (como se citó en Goodman, 2014).

Destaca también la propuesta del empresario mexicano Carlos Slim, quien propone una semana laboral de tres días y 33 horas de trabajo como fórmula para reducir el desempleo. Además, se ha mostrado partidario de retrasar la edad de jubilación, «porque retirarse a los 62 años hace insostenible el sistema» (Bolland, 17 de octubre de 2015). En su opinión, la semana laboral de tres días permitiría dar cabida a mucho más empleo permitiendo con esto «que el que quiera pueda tener dos trabajos» (Bolland, 17 de octubre 2015).

Con respecto a lo propuesto, Chierotti (9 de marzo de 2017) considera que esta medida además de «loca» es difícil de aplicar. Al evaluar los cambios en la rutina laboral de su propia empresa, explica que encontró serios problemas a la hora de reducir a tres días la jornada, optando por eliminar solo los viernes, experimento que duró tres meses. Mediante los resultados se determinó que los métodos de Slim no son sostenibles para un pequeño empresario (como lo es el propio Chierotti), pero que sí le permitieron aprender cuatro buenas lecciones: 
1. La importancia de establecer límites: establecer límites entre su tiempo personal y comercial es imperativo para mantener su cordura.

2. Trabajando de forma más inteligente, no más difícil: con menos tiempo para completar proyectos, naturalmente creará soluciones mejores y más eficientes.

3. Mantener las prioridades: se pierde tanto tiempo en detalles innecesarios. Apégate a las prioridades que impulsan el progreso del negocio para lograr el éxito final.

4. Compromiso del equipo: para que cualquier cambio drástico tenga éxito, es necesario la aceptación de los empleados y socios. Pon a todos a bordo. (Chierotti, 9 de marzo de 2017)

Desde la percepción de los trabajadores (de la muestra) los temores están vinculados a que no se respete el sueldo correspondiente al tiempo completo que vienen trabajando. Es así que $60 \%$ considera que se reducirán sus ingresos; $26 \%$ que aumentarán sus gastos de movilidad; y 5\% que aumentará el desempleo. Asimismo, existe el riesgo de que con menos tiempo para desarrollar la misma cantidad de trabajo que realiza en una jornada de ocho horas podría aumentar el estrés.

\section{Discusión}

En las últimas décadas el Perú es escenario de los cambios derivados del avance tecnológico, especialmente de la tecnología digital. Se espera el reemplazo del trabajador por la máquina, lo que originará grandes mutaciones en la demanda laboral en términos de nuevas calificaciones y cantidad de trabajadores, así como también en la forma de cómo organizar la producción y la gestión del tiempo de trabajo. Esto impactará a la sociedad.

Por otro lado, la población mantiene su ritmo de crecimiento alrededor del $1 \%$ anual; además, el $75 \%$ de la población tiene la edad para ser trabajadores dependientes con tendencia a mantenerse en esta categoría. La misma situación se presenta en otros países de América Latina. Es el caso de Chile, Weller (2007) resalta la importancia de los trabajadores jóvenes dependientes porque los asalariados adultos representan solamente dos tercios de los 
ocupados en Chile, alrededor de la mitad en Ecuador y menos de la mitad en el Perú.

La movilidad en el mercado de trabajo se está incrementando como efecto de la alta tecnificación de los procesos productivos. Se presentan las entradas y salidas de trabajadores hacia y desde empresas formales y la duración del desempleo se ha incrementado. Esto será cada vez más importante en virtud de la transformación digital y el rápido cambio tecnológico que está experimentando la producción y que impactará en las formas de organización social como la familia, entre otras.

La OCDE (2018) señala que casi la mitad de todos los empleos corren un alto riesgo de automatización y que también existe el riesgo que el desempleo aumente entre otros cambios. Los planteamientos teóricos y las políticas de empleo también muestran ese dinamismo. Esto evidencia que el mercado de trabajo está cambiando. En la década de los 90 la estrategia de empleo de la OCDE hacía hincapié en la flexibilidad laboral como respuesta al desempleo generalizado y en el 2000 se promovió diferentes mezclas de políticas públicas para fomentar el empleo para todos.

La estrategia de empleo de la OCDE, proporciona un marco integral de política para impulsar más empleos, y cualitativamente mejores en un mercado laboral más incluyente y resiliente. Se requiere diseñar e implementar una política preventiva que involucre una educación de los jóvenes para el empleo en una era de cambio digital.

Por otra parte, Weller (2007) afirma el fracaso de la flexibilización del mercado de trabajo, que no es lo mismo que la flexibilización de la jornada laboral. En los países en que se ha aplicado la flexibilización del mercado de trabajo, el desempleo y el subempleo siguen siendo problemas pendientes de solución, sin embargo, al mismo tiempo se incrementan las tasas de ganancia de los inversionistas, los niveles de inequidad social con alto impacto en la calidad de vida de los/as trabajadores/as.

Sucede lo contrario en Alemania, donde los trabajadores poseen jornadas laborales de 35 horas semanales (con 24 días de vacaciones pagadas) mostrando una alta productividad. Según Méndez (5 de marzo de 2019) en 
Alemania las jornadas laborales se respetan escrupulosamente, de forma legal se establecen normas para prohibir la recepción de correos electrónicos relacionados con temas de trabajo después que ha terminado la jornada. Pero también no está permitido en el trabajo el uso de Facebook, el uso del teléfono móvil para asuntos privados, tampoco el uso del correo electrónico para temas personales y, por supuesto, no está permitido hablar con el resto de compañeros sobre asuntos ajenos al trabajo en horario laboral.

El caso de Alemania es un ejemplo a seguir ya que focaliza las tareas y se respeta íntegramente el tiempo que le pertenece a cada labor. Por ello, cuando es hora de trabajar, es hora de trabajar. Para la implementación de esta estrategia se demanda una nueva cultura del trabajo y respeto al tiempo de vida del otro, coincidiendo con los planteamientos de Sharon (diciembre de 2015).

Una cultura del trabajo que premia la alta cantidad de horas en un puesto laboral, la falta de habilidades de planificación de los líderes, la poca claridad de las metas que las personas tienen en las funciones que desempeñan, y una relación laboral tensada por el discurso permanente de «los buenos» $\mathrm{y}$ «los malos» (elija usted quién es quién), nos llevan a trabajar más de la cuenta, producir menos de lo necesario y perjudicar tanto el crecimiento de las organizaciones como del país.

En función de estos condicionantes, la propuesta es flexibilizar la jornada y el horario laboral o una combinación de ambos, las empresas y otras instituciones deberán cambiar las condiciones laborales para facilitar que las personas combinen el trabajo, la atención a la familia y otras responsabilidades sociales. Este nuevo entorno laboral de mayor equidad y equilibrio entre el trabajo y la familia reducirá las brechas de género y disminuirá el riesgo de pobreza y exclusión.

Se plantea respetar al trabajador en su rol dentro de la sociedad, una vida que comparte entre la familia y su centro de trabajo, un espacio de trabajo en el que tiene derecho a vivir como en familia, lo que sin duda genera altos márgenes de libertad propicia para la innovación; eso guarda relación con la flexibilidad laboral, cuestionando de esta manera los horarios de trabajo y la gestión del tiempo rígido. 
La gestión del tiempo de trabajo rígido corresponde a una época donde se sostenía que un empleado no puede trabajar bien si no tiene un horario definido, es parte de nuestra experiencia, de 9 de la mañana a 9 de la noche, y de marcar una tarjeta para dejar constancia de la presencia del trabajador. Las reglas de permanencia en el trabajo eran casi inflexibles, los retrasos de 5 o 10 minutos siempre causaban molestias, así como el pedir demasiados permisos para asuntos personales durante unas horas al día. Todo ello se ha afincado con demasiada fuerza en el subconsciente laboral y en la forma de dirigir negocios, pero todo queda de lado al optar por un nuevo modelo de gestión del tiempo.

Se recomienda también fortalecer los mecanismos generadores de un clima laboral que tenga como eje central la confianza mutua dentro de la empresa o la organización, base para adoptar el modelo de gestión del tiempo flexible. Relaciones laborales basadas en la confianza pueden aumentar la productividad y dar muy buenos resultados, porque el empresario confía en que los trabajadores cumplirán los acuerdos contraídos y los trabajadores confiarán en cada uno de los miembros del equipo. Pero también al mismo tiempo supone tener la seguridad de la estabilidad y la seguridad que otorgan las normas, las estructuras formales producidas y legitimadas socialmente.

Deben quedar atrás reducir o reemplazar los sistemas de control del tiempo personal inflexibles, que significan para el trabajador quedarse día a día en la oficina. De nada sirve obligar a que el empleado permanezca de 9 a 9 delante de la computadora si en las tres últimas horas de la jornada ya está agotado y su productividad baja sustantivamente y lo que puede aportar es poco.

Es conveniente cambiar el horario rígido para otorgar ciertos grados de libertad a los trabajadores, esta acción causa un impacto positivo en el clima laboral que es una condición para lograr un mayor rendimiento en el trabajo, porque desaparece el miedo al castigo y al descuento genera confianza y conductas con menor miedo al riesgo. Crea la predisposición en cada trabajador para hablar de temas que potencialmente pueden provocar desaprobación y rechazo, este tipo de comportamiento muchas veces dio origen a una nueva invención tecnológica y ha supuesto un cambio de paradigma para la forma de desenvolverse de las personas en su trabajo. 
Como señala Abadía (2018, p. 1), «nuestro comportamiento en el ámbito laboral no es más que una prolongación o expresión de nuestra actitud ante la vida». Si tenemos confianza, seguridad de no ser despedido, de tener un buen salario y cumplir con un horario flexible, nada podrá impedir que sea cada día más productivo.

\section{Referencias}

Abadía, F. (2018). Tu actitud es tu llave maestra. Y cómo ésta puede abrirte todas las puertas. Barcelona: Gestión 2000. Recuperado de https://www.planetadelibros.com/libro-tu-actitud-es-tu-llave-maestra/280586

Arancibia, F. (2011). Flexibilidad laboral: elementos teórico-conceptuales para su análisis. Revista Ciencias Sociales, 26, 39-55. Universidad Arturo Prat., Tarapacá-Chile. Recuperado de http://www.redalyc.org/pdf/ 708/70822578003.pdf

Bolland, E. (17 de octubre de 2015). Slim propone trabajar 33 horas y tres días por semana para reducir el paro. El País. Recuperado de https://elpais.com/economia/2015/10/16/actualidad/ 1444996426_279778.html

Cage, J. M. (2016). TEORÍA DE PETER DRUCKER: «INNOVACIÓN... INNOVACIÓN... ¡SÓLO INNOVACIÓN!» Recuperado de http://ignius.com.mx/teoria-peter-drucker-innovacion-innovacion-solo-innovacion/

Casero, J. L. (2 de mayo de 2016). Equipos \& talentos (mensaje en un blog). Recuperado de https:// www.equiposytalento.com/noticias/2016/05/02/la-racionalizacion-de-horarios-es-un-factor-clave-paraaumentar-la-eficacia-y-la-productividad-en-el-trabajo

Chierotti, L. (9 de marzo de 2017). Increase Productivity Working ONLY 3 Days a Week? When Carlos Slim, Mexico's richest man, proposed a 3-day workweek, I thought I'd give it a try. Here's what I learned. Recuperado de https://www.inc.com/logan-chierotti/should-america-follow-carlos-slim-and-adopt-a-3-dayworkweek.html

Constitución Política del Perú. (1993). Recuperado de http://spij.minjus.gob.pe/content/publicaciones_oficiales/ img/Constitucion-Politica-2016.pdf

Datosmacro.expansion.com (2018). El salario mínimo sube en Rusia. Recuperado de https:/l datosmacro.expansion.com/smi/rusia

El Peruano. (1996). Decreto Legislativo N 854 Ley de Jornada de Trabajo, Horario y Trabajo en Sobretiempo. Recuperado de http://www.leyes.congreso.gob.pe/Documentos/DecretosLegislativos/00854.pdf

Gestión. (9 de mayo 2019). El futuro son los «empleados digitales» y la semana laboral de tres días. Recuperado de https://gestion.pe/tecnologia/futuro-son-empleados-digitales-semana-laboral-tres-dias-266288-noticia/

Goodman, M. (2014). Trabajar menos horas, ¿incrementa la productividad? BBC Capital. Recuperado de https:/ /www.bbc.com/mundo/noticias/2014/08/140801_vert_cap_productividad_trabajar_menos_horas_yv

Instituto Nacional de Estadística e Informática - INEI. (2018). Compendio Estadístico 2018. Recuperado de https://www.inei.gob.pe/media/MenuRecursivo/publicaciones_digitales/Est/Lib1635/compendio2018.html.

La Información (4 de enero de 2017). Un experimento en Suecia sugiere que la jornada reducida es costosa para las empresas. Recuperado de https://www.lainformacion.com/economia/experimento-Suecia-afirmatrabajar-seis-horas-no-funciona_0_987201624.html 
Menco, D. (marzo de 2008). La nueva economía clásica y el mercado laboral. Contribuciones a la Economía. Recuperado de http://www.eumed.net/ce/2008a/dmr.htm

Méndez, F. (5 de marzo de 2019). Business ¿Por qué los alemanes trabajan menos, pero producen más? Recuperado de http://forbes.es/business/5682/por-que-los-alemanes-trabajan-menos-pero-producen-mas/

Ministerio de la Producción. (2017). Este año se crearían más de 26 mil micro y pequeñas empresas (mype) lideradas por emprendedoras, Lima. Recuperado de https://www.produce.gob.pe/index.php/k2/noticias/item/ 243-produce-el-peru-contara-este-ano-con-1-millon-200-mil-mujeres-emprendedoras

Moncat.gencat.cat (2019). Generalitat de Catalunya-gencat.cat (mensaje en un blog). Jornada vacacione y permisos. Recuperado de http://moncat.gencat.cat/es/franca/informacio-sobre-tramits/informacio-laboralbasica/jornada-vacances-ipermisos

Moncat.gencat.cat (2019). Generalitat de Catalunya-.gencat.cat (mensaje en un blog). Jornada vacaciones y permisos. Recuperado de http://moncat.gencat.cat/es/franca/informacio-sobre-tramits/informacio-laboralbasica/jornada-vacances-ipermisos

Opere, M. (2019). Blog sobre retención y desarrollo del capital humano. Cómo calcular la productividad de los empleados (Mensaje en un blog). Recuperado el 22 de octubre 2019 de https://blog.grupo-pya.com/ calcular-la-productividad-los-empleados/

Organización Internacional del Trabajo - OIT. (2019). Alternativas que pueden facilitar la conciliación de vida laboral y familiar. Notas OIT. Trabajo y Familia, 5. Recuperado de https://www.lo.org/wcmsp5/groups/publicl —americas/-ro-lima/documents/publication/wcms_189334.pdf

Organización para la Cooperación y el Desarrollo Económico - OCDE. (2015). El Futuro de la Productividad, Nota conjunta de política del Departamento de Asuntos Económicos y de la Dirección de Ciencia, Tecnología e Innovación. Recuperado de http://www.oecd.org/economy/growth/El-futuro-de-laproductividad.pdf

Organización para la Cooperación y el Desarrollo Económico - OCDE. (2018). La productividad con un trabajo más eficiente en lugar de «más duro». Recuperado de https://www.elboletin.com/tag/productividad

Pozen, R. (2012). Productividad extrema. Recuperado de https://resumido.com/es/libro.php/736/productividadextrema

Sharon, I. (diciembre de 2015). HSEC Productividad v/s jornadas laborales. Recuperado de http://www.emb.cl/ hsec/articulo.mvc?xid=815\&edi=36\&xit=productividad-v/s-jornadas-laborales

Weller, J. (2007). La flexibilidad del mercado de trabajo en América Latina y el Caribe. Aspectos del debate, alguna evidencia y políticas. CEPAL-SERIE Macroeconomía del desarrollo, 61. 